\title{
Regeneration of 5-HT fibers in hippocampal heterotopia of methylazoxymethanol-induced micrencephalic rats after neonatal 5,7-DHT injection
}

\author{
Arata Nakamura · Taro Kadowaki • \\ Shin-ichi Sakakibara $\cdot$ Kanji Yoshimoto · \\ Koichi Hirata $\cdot$ Shuichi Ueda
}

Received: 2 April 2009/Accepted: 8 June 2009/Published online: 7 July 2009

(C) Japanese Association of Anatomists 2009

\begin{abstract}
In order to elucidate the regeneration properties of serotonergic fibers in the hippocampus of methylazoxymethanol acetate (MAM)-induced micrencephalic rats (MAM rats), we examined serotonergic regeneration in the hippocampus following neonatal intracisternal 5,7dihydroxytryptamine (5,7-DHT) injection. Prenatal exposure to MAM resulted in the formation of hippocampal heterotopia in the dorsal hippocampus. Immunohistochemical and neurochemical analyses revealed hyperinnervation of serotonergic fibers in the hippocampus of MAM rats. After neonatal 5,7-DHT injection, most serotonergic fibers in the hippocampus of 2-week-old MAM rats had degenerated, while a small number of serotonergic fibers in the stratum lacunosum-moleculare (SLM) of the hippocampus and in the hilus adjacent to the granular cell layer of the dentate gyrus (DG) had not. Regenerating serotonergic fibers from the SLM first extended terminals into the hippocampal heterotopia, then fibers from the hilus reinnervated the DG and some fibers extended to the heterotopia. These findings suggest that the hippocampal heterotopia exerts trophic target effects for regenerating
\end{abstract}

A. Nakamura · T. Kadowaki $\cdot$ K. Hirata

Department of Neurology, Dokkyo Medical University

School of Medicine, Mibu, Tochigi 321-0293, Japan

S. Sakakibara $\cdot$ S. Ueda $(\square)$

Department of Histology and Neurobiology,

Dokkyo Medical University School of Medicine,

Mibu, Tochigi 321-0293, Japan

e-mail: shu-ueda@dokkyomed.ac.jp

K. Yoshimoto

Department of Legal Medicine,

Kyoto Prefectural University of Medicine,

Kyoto, Japan serotonergic fibers in the developmental period in micrencephalic rats.

Keywords Methylazoxymethanol acetate ·

Micrencephalic rat · Regeneration .

Hippocampal heterotopia $\cdot$ Serotonergic fibers

\section{Introduction}

Serotonergic fibers in the adult mammalian nervous system display a remarkable capacity for regeneration after selective chemical and non-selective traumatic injury (Azmitia et al. 1978; Björklund and Stenevi 1979; Zhou and Azmitia 1986; Ueda and Kawata 1994). Serotonergic fibers can survive such injuries and, with time, send back axons to reinnervate denervated regions (Azmitia et al. 1978; Zhou and Azmitia 1986; Haring 1991). At 8-12 weeks after intracerebroventricular and intracerebral injection of a specific neurotoxin, 5,6- or 5,7-dihydroxytryptamine (DHT), the forebrain regions in adult rats receive varying densities of regenerating serotonergic fibers (Björklund and Stenevi 1979; Jacobs and Azmitia 1992; Ueda and Kawata 1994). In contrast, neonatal intracisternal 5,7-DHT injection induces long-lasting serotonin depletion without evidence of regeneration in regions of the forebrain, including the hippocampal formation (Towle et al. 1984; Volpe et al. 1992; Pranzatelli and Martens 1992; Ueda et al. 2005).

Methylazoxymethanol acetate (MAM) is an antimitotic agent that interferes with nucleic acid methylation (Johnston and Coyle 1982). Exposure of pregnant rats to MAM at gestational day 15 results in micrencephaly with cortical, periventricular and hippocampal heterotopias in offspring (Chvassus-Au-Louis et al. 1998), as well as alteration of neurotransmitter systems in the brain (Johnston and Coyle 
1982; Catabeni and Di Lica 1997). All of these heterotopias in the MAM-induced micrencephalic rat (MAM rat) receive excessive catecholaminergic, serotonergic and $\gamma$-aminobutyric acid (GABA) innervations (Johnston and Coyle 1982; Catabeni and Di Lica 1997; Baraban et al. 2000; Watabe et al. 2005). Electrophysiological and neuroanatomical analyses have demonstrated that heterotopic neurons in the hippocampus display cellular properties of neocortical neurons with altered fiber connections (Chvassus-Au-Louis et al. 1998; Colacitti et al. 1998) and atypical electrophysiological characteristics because of aberrant expression of ion channels and transmitter receptors (Rafiki et al. 1998; Castro et al. 2001). These findings suggest a possible change in plasticity in the hippocampal heterotopias of MAM rats. Although impaired synaptic plasticity has been reported in the hippocampus of MAM rats (Ramakers et al. 1993), the characteristics of such regeneration have not been determined. We therefore neurochemically and immunohistochemically examined the regeneration of serotonergic fibers in regions of the hippocampal formation, particularly in the dorsal hippocampus of MAM rats following neonatal intracisternal administration of 5,7-DHT.

\section{Materials and methods}

\section{Animals}

Pregnant Sprague-Dawley (SD) rats (Japan Charles River Laboratories, Tsukuba, Japan) were intraperitoneally injected with either $25 \mathrm{mg} / \mathrm{kg}$ MAM $(10 \mathrm{mg} / \mathrm{ml}$ physiological saline) or an equal volume of vehicle alone at gestational day 15 (Ueda et al. 1999; Watabe et al. 2005). At 3 days after birth, pups initially underwent intraperitoneal injection of desipramine (DMI; Sigma, St. Louis, MO; $20 \mathrm{mg} / \mathrm{kg}$ ), a catecholamine uptake inhibitor. After $1 \mathrm{~h}$, each pup was injected intracisternally with 5,7-DHT (75 $\mu \mathrm{g}$ of free base in $10 \mu \mathrm{l}$ of saline with $0.1 \%$ ascorbic acid) or $10 \mu \mathrm{l}$ of saline with $0.1 \%$ ascorbic acid under hypothermic anesthesia (Ueda et al. 2005). Pups were returned to animal cages and biological foster dams for 4 weeks. Offspring were weaned at 4 weeks of age, and rats of both sexes were used in the present study. All animals were maintained with ad libitum access to food and water. All procedures in this study were approved by the Animal Care and Use Committee of Dokkyo Medical University School of Medicine.

Animals were divided into four groups: control (sham) group, 5,7-DHT-injected (DHT), MAM-injected (MAM) group and MAM plus 5,7-DHT-injected (MAM + DHT) group. Similar to previous reports (Towle et al. 1984; Volpe et al. 1992; Pranzatelli and Martens 1992; Ueda et al. 2005), current neurochemical analyses did not reveal any evidence of 5-HT regeneration in the DHT group (see "Results"). Rats from the MAM and MAM + DHT groups were thus used for immunohistochemical analysis.

\section{Biochemical measurements}

Biochemical measurements using high-performance liquid chromatography (HPLC) were performed as described previously (Ueda et al. 1999; Watabe et al. 2005). Briefly, at age 14 weeks, rats ( $n=5-6$ in each group) were killed with a lethal overdose of sodium pentobarbital and decapitated. Brains were immediately removed and washed with ice-cold $0.1 \mathrm{M}$ phosphate-buffered saline (PBS; pH 7.4), and the dorsal half of the hippocampus was rapidly dissected and frozen in liquid nitrogen. Tissues were weighed, homogenized in $0.1 \mathrm{M}$ perchloric acid containing $0.1 \mathrm{mM} \mathrm{Na} \mathrm{N}_{2}$-ethylenediamine tetraacetate and then filtered. Filtered homogenates were centrifuged at 15,000 rpm for $20 \mathrm{~min}$. Supernatants were analyzed for serotonin (5-HT), 5-hydroxyindolacetic acid (5-HIAA) and noradrenalin (NA) by HPLC with electrochemical detection (Shimazu Co., Kyoto, Japan). Results were statistically compared using Student's two-tailed $t$ test.

Tissue preparation

At $2,4,5$ or 14 weeks after birth, rats $(n=4-7$ in each group) were killed with a lethal overdose of sodium pentobarbital and were transcardially perfused with physiological saline, followed by $500 \mathrm{ml}$ of a mixture of $4 \%$ paraformaldehyde in $0.1 \mathrm{M}$ PBS. Each rat brain was immediately removed and post-fixed in the same fixative for $48 \mathrm{~h}$ at $4^{\circ} \mathrm{C}$, and then immersed in $30 \%$ sucrose solution for $48 \mathrm{~h}$. Brains were frozen, and serial sections $(30 \mu \mathrm{m})$ were prepared using a cryostat. Sections were collected in $0.1 \mathrm{M}$ PBS containing $0.3 \%$ Triton-X 100, and incubated in $1 \% \mathrm{H}_{2} \mathrm{O}_{2}$ solution treated with $1.5 \%$ normal goat serum or normal horse serum at room temperature for $1 \mathrm{~h}$ to prevent non-specific staining. Sections were then incubated with primary antibodies, polyclonal anti-serotonin antiserum (1:10,000 dilution; Ueda et al. 1999; Watabe et al. 2005) for 4 days at $4^{\circ} \mathrm{C}$. After rinsing several times in PBS, sections were processed for avidinbiotin peroxidase immunostaining and then reacted with $20 \mathrm{mg}$ of $3,3^{\prime}$-diaminobenzidine (DAB) in $100 \mathrm{ml}$ of $0.05 \mathrm{M}$ Tris- $\mathrm{HCl}$ buffer ( $\mathrm{pH} 7.4$ ), containing $20 \mu \mathrm{l}$ of $30 \% \mathrm{H}_{2} \mathrm{O}_{2}$ for $10 \mathrm{~min}$. Sections were mounted on gelatincoated slides and air-dried. Some sections were stained with cresyl-violet in order to identify the cytoarchitectonic area of the hippocampus and were then dehydrated in ethanol, cleared in xylene and coverslipped with Entellan. 
Optical density analysis

Serotonin-immunostained sections were analyzed semiquantitatively using NIH image (free software). Mean optical density (OD) of serotonin-immunostained sections was measured in the hippocampal heterotopia. In this analysis, the OD of the empty lumen of blood vessels was used to determine relative OD. Results were analyzed using analysis of variance (ANOVA), and post-hoc comparisons were based on Sheffe's $F$ test using a significance level of 0.05 .

\section{Results}

\section{General morphology}

In all MAM-injected groups, Nissl-stained sections exhibited cortical malformations consisting of a thinner neocortex than in the control and DHT groups, as well as heterotopias in the CA1 field of the hippocampus (hippocampal heterotopia) (Fig. 2a), in the periventricular region near the caudate putamen (periventricular heterotopia) and in the deep cortical layers (cortical heterotopia) (Johnston and Coyle 1982; Catabeni and Di Lica 1997; Chvassus-AuLouis et al. 1998). No differences in morphology were observed between the MAM and MAM + DHT groups in Nissl-stained sections.

\section{Biochemical measurements}

Concentrations of 5-HT and 5-HIAA were determined by HPLC analysis in the hippocampus of the control, DHT, MAM and MAM + DHT groups. The results are summarized in Table 1. Concentrations of NA, 5-HT and 5-HIAA

Table 1 Concentrations (pM/ng) of NA, 5-HT and 5-HIAA in hippocampus of various experimental groups

\begin{tabular}{llll}
\hline $\begin{array}{l}\text { Experimental } \\
\text { groups }\end{array}$ & NA & 5-HT & 5-HIAA \\
\hline Control (sham) & $1.98 \pm 0.18$ & $1.74 \pm 0.19$ & $1.95 \pm 0.02$ \\
DHT & $1.86 \pm 0.27$ & ND & ND \\
MAM & $3.69 \pm 0.47^{*, \#}$ & $2.94 \pm 0.29^{*}$ & $1.98 \pm 0.14$ \\
MAM + DHT & $2.45 \pm 0.32$ & $0.12 \pm 0.04^{*, \dagger}$ & $0.54 \pm 0.35^{*, \dagger}$ \\
\hline
\end{tabular}

All values are the mean \pm SEM

$N D$ Not detected, MAM methylazoxymethanol acetate-injected group, DHT 5,7-dihydroxytryptamine-injected group, $M A M+D H T$ methylazoxymethanol acetate plus 5,7-dihydroxytryptamine-injected group, $N A$ noradrenalin, 5-HT 5-hydroxytryptamine (serotonin), 5-HIAA 5-hydroxyindolacetic acid

${ }^{*} P<0.05$ versus control group; ${ }^{\#} P<0.05$ versus DHT group; ${ }^{\dagger} P<0.05$ versus MAM group tended to increase in the hippocampus of the MAM group as compared with other groups. Concentrations of 5-HT and 5-HIAA in the hippocampus of the DHT and MAM + DHT groups were reduced, with a significant difference noted between the MAM and MAM + DHT groups (Table 1, $P<0.05)$.

\section{5-HT immunohistochemistry}

As with control (sham) rats (Fig. 1), a highly laminar pattern of distribution of serotonergic fibers was noted in the hippocampus and overlying cortex of MAM groups rats, which is consistent with our previous findings (Ueda et al. 1999; Watabe et al. 2005). The density of serotonergic fibers was highest in the stratum lacunosum-moleculare (SLM) of CA1, the stratum oriens and stratum radiatum (SR) of $\mathrm{CA} 3$ and the heterotopias, lower in the molecular layer (ML) of the dentate gyrus (DG) and lowest in the pyramidal cell layer (PCL) of CA3 and the granule cell layer (GCL) of the DG (Fig. 2b, c, d). In the hilus of MAM rats, a dense laminar distribution of serotonergic fibers was identified immediately subjacent to the GCL (sub-GCL) (Fig. 2c, arrow). All of these serotonergic fibers were varicose fibers with varicosities of various sizes.

Most serotonergic fibers in the hippocampus had disappeared in 2-week-old (2W) rats from the MAM + DHT group (Fig. 2e). Only a small number of surviving serotonergic fibers was observed in the SLM of CA1, SR and PCL of CA3 (Fig. 2, arrows) and in the hilus immediately adjacent to the GCL (Fig. $2 \mathrm{~g}$, arrows). At this stage, hippocampal heterotopia was completely devoid of serotonergic fibers, whereas surrounding areas contained a few serotonergic fibers (Fig. 2h, arrow).

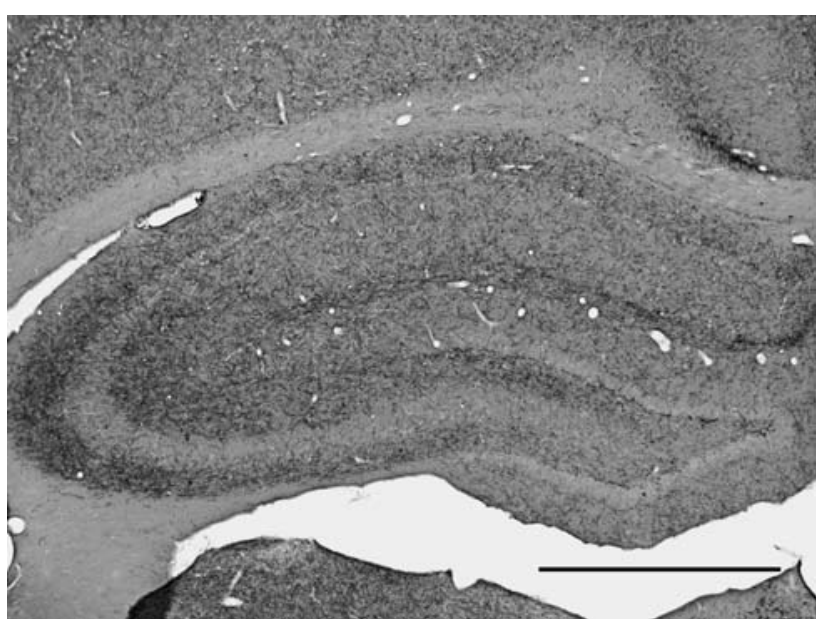

Fig. 1 A photomicrograph of coronal sections from the dorsal hippocampus of 14-week-old control (sham) rat after 5-HT immunohistochemical staining. A highly laminar pattern of distribution of serotonergic fibers is evident. Bar $1 \mathrm{~mm}$ 
Fig. 2 Photomicrographs of coronal sections from the dorsal hippocampus of 14-week-old MAM-induced micrencephalic rat (a-d) and 2-week-old neonatal 5,7-DHT-injected MAM-induced micrencephalic rat $(\mathbf{e}-\mathbf{h})$. Arrows Indicate hippocampal heterotopia $(\mathbf{a}, \mathbf{b}, \mathbf{e})$. a Stained with cresyl-violet. b-d After 5-HT immunohistochemistry. c Highmagnification view of dentate gyrus. A dense plexus of serotonergic fibers is evident in the hilus subjacent to the granule cell layer (GCL) (arrow). d High-magnification view of hippocampal heterotopia. Serotonergic fibers with varicosities of various sizes are observed within the heterotopia. $\mathbf{e}-\mathbf{h}$ Photomicrographs showing 5-HT immunohistochemistry counterstained with cresylviolet. High-magnification view of CA3 (f), dentate gyrus (g) and heterotopia (h). A few serotonergic fibers have survived (arrows). GCL Granule cell layer, $P C L$ pyramidal cell layer. Bar $200 \mu \mathrm{m}(\mathbf{a}, \mathbf{b}, \mathbf{e}) ; 50 \mu \mathrm{m}$ $(\mathbf{c}, \mathbf{d}, \mathbf{f}, \mathbf{g}, \mathbf{h})$
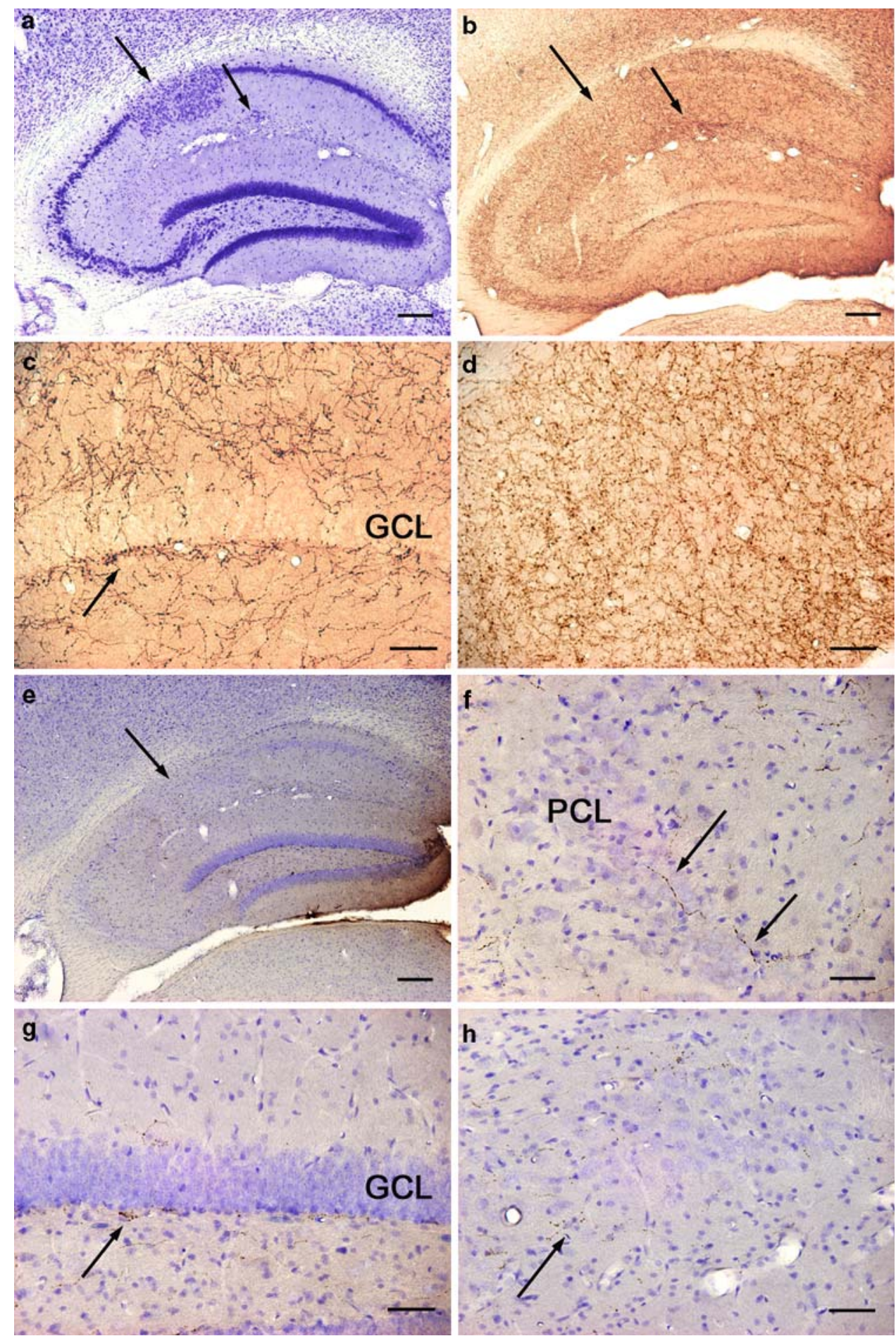

Hippocampal heterotopias of $4 \mathrm{~W}$ rats from the MAM + DHT group were endowed with three different types of serotonergic fibers based on morphological criteria: fine fibers characterized by a thin caliber axon and small fusiform varicosities (Fig. 3a, b, arrows); distorted thick fibers with a thick caliber (Fig. 3g); beaded fibers characterized by large, spherical varicosities connected by thin intervaricose segments (Fig. 3a-c, arrowheads). Fine fibers and distorted thick fibers were mainly distributed around the heterotopias, while beaded fibers were located within the heterotopia in addition to the surrounding area. All types of fibers traced laterally to the SLM of CA3 (Fig. 3b). Some of the fine fibers and distorted thick fibers entered the heterotopia, and extended collaterals that showed a beaded-shape within the heterotopia. At this stage, only a few serotonergic fibers were observed in the 
Fig. 3 Photomicrographs of coronal sections from the dorsal hippocampus of 4-week-old (a-d) and 14-week-old (e-h) neonatal 5,7-DHT-injected MAM-induced micrencephalic rat. a High magnification view of CA1 region and heterotopia $(H)$. Hippocampal heterotopia is delineated by dotted lines. Serotonergic fine fibers with small spherical varicosities (arrow) and serotonergic beaded fibers with large varicosities (arrowheads) are seen in the area surrounding the heterotopia. Some of these fibers penetrate into the heterotopia. b Highmagnification view of stratum lacunosum-moleculare. Serotonergic fine fibers with small spherical varicosities (arrows) and serotonergic beaded fibers with large varicosities (arrowhead) can be traced from the SLM to the heterotopia. c Highmagnification view of figure $\mathbf{a}$. Serotonergic beaded fibers with large varicosities (arrowhead) are evident. d Highmagnification view of the dentate gyrus. Serotonergic fibers have survived in the hilus adjacent to the granule cell layers (GCL). e Stained with cresyl-violet. Arrows Indicate hippocampal heterotopias.

f-h Stained by 5 -HT

immunohistochemistry.

f Hippocampal heterotopias are densely innervated by serotonergic fibers (arrows). g Area surrounding the heterotopia contains a mixed population of fine, beaded and thick, distorted serotonergic fibers, while beaded fibers predominate in the heterotopia $(H)$. h Serotonergic beaded fibers are present in the molecular layer throughout the GCL (arrows). Bar $50 \mu \mathrm{m}(\mathbf{a}, \mathbf{h}) ; 25 \mu \mathrm{m}$ (b-d, g); $200 \mu \mathrm{m}(\mathbf{e}, \mathbf{f})$
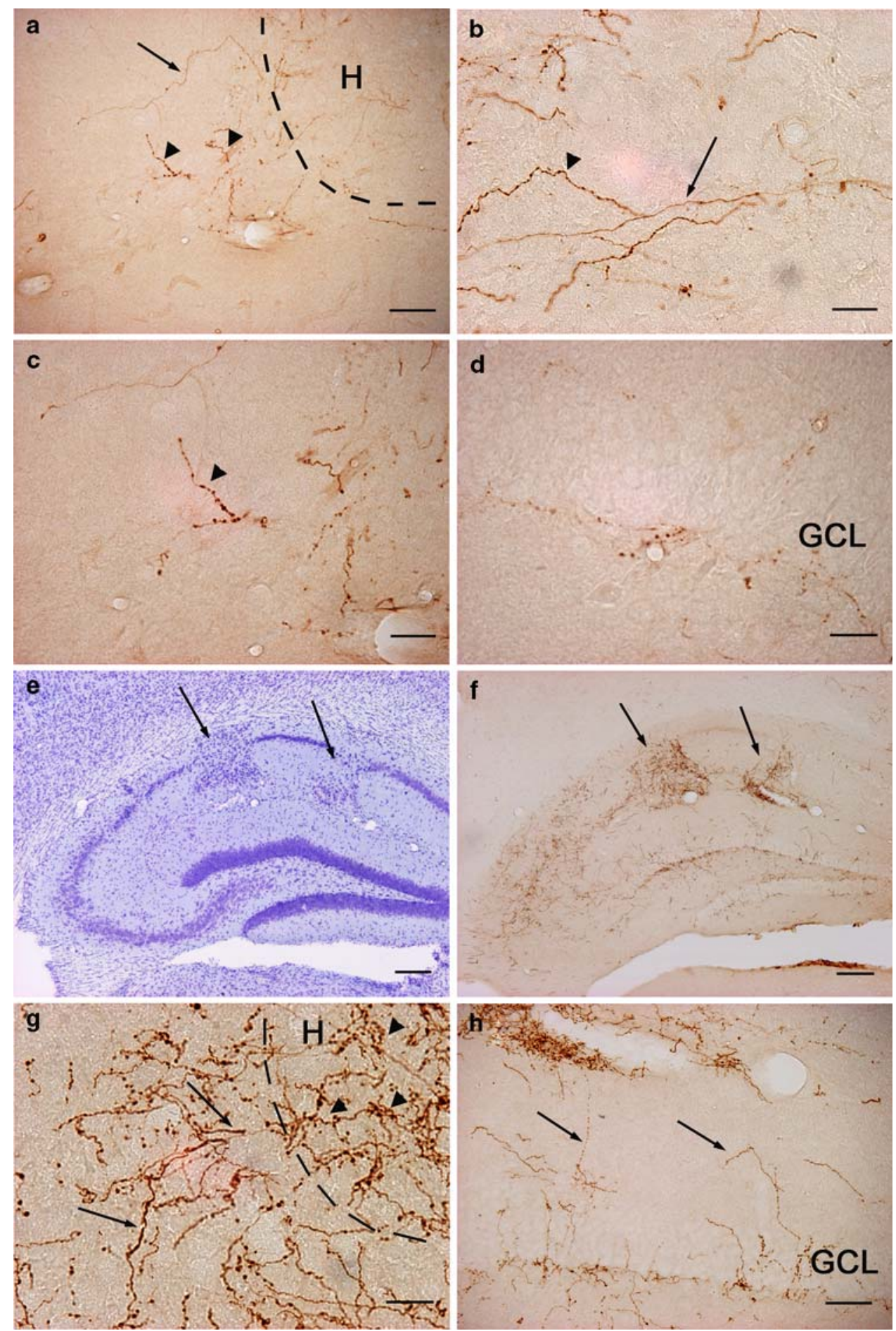

sub-GCL (Fig. 3d). However, the number of these fibers had increased in $5 \mathrm{~W}$ rats, and these fibers traversed the GCL and extended into the ML of the DG.

The hippocampal formation in $14 \mathrm{~W}$ rats of the MAM + DHT group exhibited a regional specific reinnervation of serotonergic fibers. However, the density of fibers remained less than that seen in the MAM group.
A large number of serotonergic fibers was seen in the hippocampal heterotopias (Fig. 3f, arrows), and most such fibers were beaded fibers. In the area surrounding the heterotopia, a small number of fine fibers intermingled with beaded fibers. Furthermore, beaded fibers emanating from thick fibers were sometimes observed within the heterotopia (Fig. 3g, arrowheads). In the DG, the serotonergic fiber 


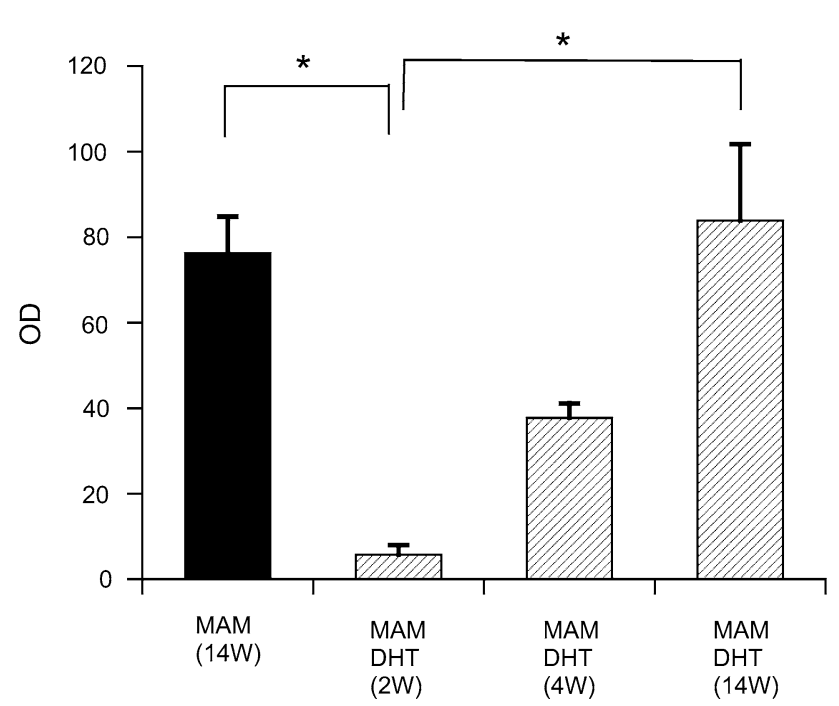

Fig. 4 Optical density of hippocampal heterotopia stained with 5HT immunohistochemistry. Optical density was measured for MAM (closed column) and MAM + DHT (hatched column) groups at 2, 4 and 14 weeks, and is given as mean \pm SEM. $P<0.05$

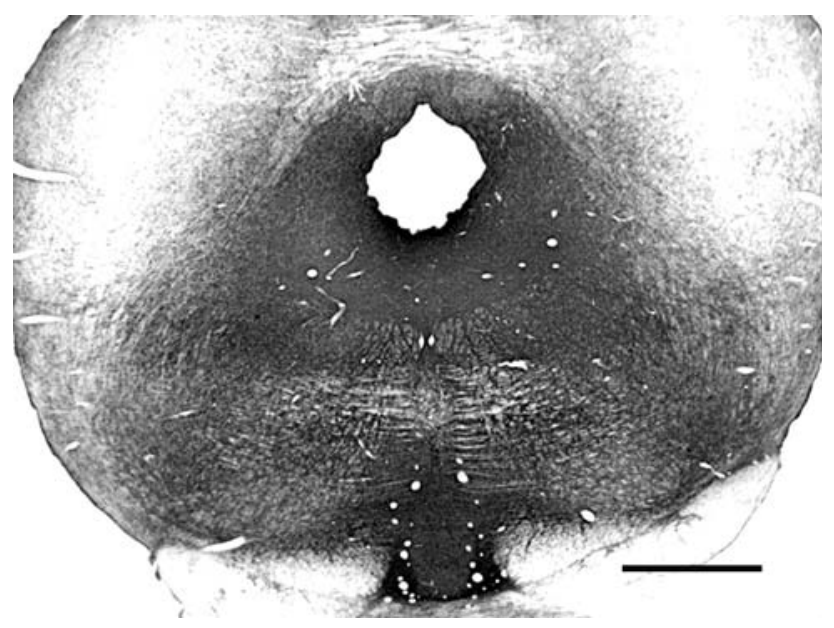

Fig. 5 Photomicrographs of coronal sections from 14-week-old MAM + DHT rats at the level of pons, including dorsal and medial raphe nuclei. Bar $1 \mathrm{~mm}$

plexus of the sub-GCL became denser in the suprapyramidal blade of the GCL than that in the infrapyramidal GCL (Fig. 3f). Serotonergic fibers traversing the GCL and ML to the heterotopias were also evident (Fig. 3h, arrows).

In quantitative analysis (Fig. 4), ANOVA revealed significant differences among the groups $(F(3,8)=12.89$, $P<0.03)$. OD reflecting the density of serotonin-immunoreactive fibers and/or terminals in the hippocampal heterotopia was significantly reduced in $2 \mathrm{~W}$ MAM + DHT group rats. OD was significantly higher in 14W MAM + DHT group rats when compared with $2 \mathrm{~W}$ MAM + DHT group rats (Fig. 4). The dorsal and medial raphe nuclei
(DRN, MRN), in which serotonergic neurons projected to the forebrain, including the hippocampus (Azmitia and Segal 1978), of 14W MAM + DHT group rats showed hyperinnervation by serotonergic regenerating fibers (Fig. 5).

\section{Discussion}

In this study, the hippocampal formation of MAM rats was found to receive abundant serotonergic innervations (Fig. 2b), as noted in previous reports (Jonsson and Hallman 1982; Ueda et al. 1999; Watabe et al. 2005). Kodama et al. (2000) reported serotonergic hyperinnervation in the cerebral heterotopia using $\left[{ }^{3} \mathrm{H}\right]$-paroxetin-binding autoradiography. The present immunohistochemical study provides the first demonstration of serotonergic hyperinnervation in the hippocampal heterotopia in MAM-induced micrencephalic rats.

Several studies have demonstrated that neurons in the hippocampal heterotopias of MAM rats are phenotypically similar to neocortical supragranular neurons (ChvassusAu-Louis et al. 1998; Castro et al. 2002; Paredes et al. 2006; Harrington et al. 2007). The hippocampal heterotopia may therefore receive serotonergic hyperinnervation similar to the cortical heterotopia.

Immunohistochemical analyses demonstrated that most serotonergic fibers in the hippocampal formation, including hippocampal heterotopia, were diminished after neonatal 5,7-DHT injection. A few serotonergic fibers in the hippocampal formation of the $2 \mathrm{~W}$ MAM + DHT group rats escaped degeneration and were observed in a very restricted area of the hippocampal formation (Fig. 2e-h). After this stage, regenerating processes of serotonergic fibers were identified in the hippocampal formation.

At the first stage of regeneration (up to $4 \mathrm{~W}$ ), surviving serotonergic fibers near the heterotopia and SLM extended terminals and/or collaterals within the heterotopia, and then (after 4W) regenerating fibers from the hilar regions entered the heterotopia through the GCL and ML. The hippocampal heterotopia in $14 \mathrm{~W}$ rats from the MAM + DHT group was densely innervated by regenerated serotonergic fibers. These immunohistochemical data were confirmed by neurochemical analyses. Intracisternal 5,7-DHT injection with DMI significantly altered concentrations of serotonin and 5-HIAA, but not that of NA (Table 1).

In agreement with previous findings (Towle et al. 1984; Volpe et al. 1992; Ueda et al. 2005), no 5-HT or 5-HIAA was detectable in the hippocampus of the DHT group (Table 1). However, HPLC revealed detectable levels of 5HT in the MAM + DHT group. Concentrations of 5-HT in 
this group decreased by $4 \%$ when compared with the MAM group (Table 1). Taken together with previous studies of neonatal 5,7-DHT injection (Towle et al. 1984; Volpe et al. 1992; Ueda et al. 2005), the present data suggest that the regenerative capacity of the serotonergic neuron system in MAM-induced micrencephalic rats after neonatal neurotoxin injury is higher than that in normal rats. The finding that the hippocampal heterotopia is the most densely reinnervated area throughout the hippocampal formation of MAM-induced micrencephalic rat suggests a trophic effect on serotonergic regeneration in the hippocampal heterotopia following neonatal intracisternal 5,7-DHT injection. The mechanisms underlying serotonergic reinnervation in MAM rats remain poorly understood. Further study is thus needed to clarify these mechanisms.

In hippocampal formation in normal rats, serotonergic fibers mostly arise from two raphe nuclei in the brainstem: the dorsal raphe nucleus (DRN) and the median raphe nucleus (MRN) (Moore and Halaris 1975; Azmitia and Segal 1978; Köhler and Steinbusch 1982; Vertes and Martin 1988; Vertes 1991). The hippocampus receives dense serotonergic innervations from both the DRN and MRN, whereas serotonergic fibers from the DRN and MRN exhibit heterogeneic distribution in the DG. The MRN projects to the stratum radiatum of $\mathrm{CA} 3$ and the regions between the stratum radiarum and the SLM of CA1. In the DG of normal rats, serotonergic fibers arising from the DRN project preferentially to the molecular layer, whereas serotonergic fibers from the MRN project mainly to the hilar regions, forming a dense plexus adjacent to the GCL (Azmitia and Segal 1978). Most serotonergic fibers in the hippocampal formation degenerate after intracisternal 5,7DHT injection, with surviving serotonergic fibers then sprouting and reorganizing within the hippocampal formation of MAM rats. The connections of serotonergic fibers between the hippocampal heterotopia and the plexuses in the SLM or hilar regions suggest the sprouting profiles of serotonergic fibers. Most regenerating serotonergic fibers in the hippocampal heterotopia may have originated from the MRN. Further tract tracing studies are needed to clarify this notion. In addition, serotonin immunohistochemistry revealed the hyperinnervation of regenerating fibers in the DRN and MRN of $14 \mathrm{~W}$ MAM + DHT group rats. Because of their high density, the cell bodies in these sections were difficult to distinguish. Thus, another serotonergic neuronal marker antibody, anti-tryptophan hydroxylase antibody, may be necessary to overcome this problem.

Acknowledgments This work was supported by a grant from the Academic Frontier Project of The Ministry of Education, Culture, Sports, Science and Technology, Japan (to S.U.).

\section{References}

Azmitia EC, Segal M (1978) An autoradiographic analysis of the differential ascending projections of the dorsal and median raphe nuclei in the rat. J Comp Neurol 179:641-668

Azmitia EC, Buchan AM, Williams JH (1978) Structural and functional restoration by collateral sprouting of hippocampal 5-HT axon. Nature 274:374-376

Baraban SC, Wenzel HJ, Hochman DW, Schwartzkrion PA (2000) Characterization of heterotopic cell clusters in the hippocampus of rats exposed to methylazoxymethanol in utero. Epilepsy Res 39:87-102

Björklund A, Stenevi U (1979) Regeneration of monoaminergic and cholinergic neurons in the mammalian central nervous system. Physiol Rev 59:62-100

Castro PA, Cooper EC, Lowenstein DH, Baraban SC (2001) Hippocampal heterotopia lack functional $\mathrm{Kv} 4.2$ potassium channels in the methylazoxymethanol model of cortical malformations and epilepsy. J Neurosci 21:6626-6634

Castro PA, Pleasure SJ, Baraban SC (2002) Hippocampal heterotopia with molecular and electrophysiological properties of neocortical neurons. Neuroscience 114:961-972

Catabeni F, Di Lica M (1997) Developmental models of brain dysfunctions induced by targeted cellular ablations with methylazoxymethanol. Physiol Rev 77:199-215

Chvassus-Au-Louis N, Rafiki A, Jorquera I, Ben-Ari Y, Represa A (1998) Neocortex in the hippocampus: an anatomical and functional study of CA1 heterotopias after prenatal treatment with methylazoxymethanol in rats. J Comp Neurol 394: $520-536$

Colacitti C, Sancini G, Franceschetti S, Catabeni F, Avenzini G, Spreafico R, Di Lica M, Battaglia G (1998) Altered connections between neocortical and heterotopic areas in methylazoxymethanol-treated rat. Epilepsy Res 32:49-62

Harrington EP, Möddel G, Najm IM, Baraban SC (2007) Altered glutamate receptor-transporter expression and spontaneous seizures in rats exposed to methylazoxymethanol in utero. Epilepsia 48:158-168

Haring JH (1991) Reorganization of the area dentate serotonergic plexus after lesions of the median raphe nucleus. J Comp Neurol 306:576-584

Jacobs BL, Azmitia EC (1992) Structure and function of brain serotonin system. Physiol Rev 7:165-229

Johnston MV, Coyle JT (1982) Cytotoxic lesions and the development of transmitter systems. Trends Neurosci 5:153-156

Jonsson G, Hallman H (1982) Effects of prenatal methylazoxymethanol treatment on the development of central monoamine neurons. Brain Res Dev Brain Res 2:513-530

Kodama Y, Nonaka R, Hagino Y, Watanabe M (2000) The distribution of serotonergic nerves in micrencephalic rats treated prenatally with methylazoxymethanol. Neurochem Res 25 : 497-501

Köhler C, Steinbusch H (1982) Identification of serotonin and nonserotonin-containing neurons of the mid-brain raphe projecting to entorhinal area and hippocampal formation. A combined immunohistochemical and fluorescent retrograde tracing study in the rat brain. Neuroscience 7:951-975

Moore RY, Halaris AE (1975) Hippocampal innervation by serotonin neurons of the midbrain raphe in the rat. J Comp Neurol 164:171-184

Paredes M, Pleasure SJ, Baraban SC (2006) Embryonic and early postnatal abnormalities contributing to the development of hippocampal malformations in a rodent model of dysplasia. J Comp Neurol 495:133-148 
Pranzatelli MR, Martens JM (1992) Plasticity and ontogeny of the central 5-HT transporter: effect of neonatal 5,7-dihydroxytryptamine lesions in the rat. Brain Res Dev Brain Res 70:191-195

Rafiki A, Chvassus-Au-Louis N, Ben-Ari Y, Khrestchatisky M, Represa A (1998) Glutamate receptors in dysplastic cortex: an in situ hybridization and immunohistochemistry study in rats with prenatal treatment with methylazoxymethanol. Brain Res 782:142-152

Ramakers GM, Urban IJA, De Graan PNE, Di Luca M, Cattabeni F, Gispen WH (1993) The impaired long-term potentiation in the CA1 field of the hippocampus of cognitive deficient micrencephalic rats is restored by D-serine. Neuroscience 54:49-60

Towle A, Breese GR, Mueller RA, Coyle S, Lauder JM (1984) Early postnatal administration of 5,7-DHT: effects on serotonergic neurons and terminals. Brain Res 310:67-75

Ueda S, Kawata M (1994) Regeneration of serotonergic immunoreactive fibers in the brain of 5,6-dihydroxytryptamine treated rat. J Hirnforsch 35:159-180

Ueda S, Nishimura A, Kusuki T, Takeuchi Y, Yoshimoto K (1999) Delayed 5-HT release in the developing cortex of micrencephalic rats. Neuroreport 10:1215-1219
Ueda S, Sakakibara S, Yoshimoto K (2005) Effect of long-lasting serotonin depletion on environmental enrichment-induced neurogenesis in adult rat hippocampus and spatial learning. Neuroscience 135:395-402

Vertes RP (1991) A PHA-L analysis of ascending projections of the dorsal raphe nucleus in the rat. J Comp Neurol 313:643-668

Vertes RP, Martin GF (1988) Autoradiographic analysis of ascending projections from the pontine and mesencephalic reticular formation and median raphe nucleus of the rat. J Comp Neurol 275:511-541

Volpe BT, Hendrix CS, Park DH, Towle AC, Davis HP (1992) Early post-natal administration of 5,7-dihydroxytryptamine destroys 5-HT neurons but does not affect spatial memory. Brain Res 589:262-267

Watabe Y, Yoshimoto K, Eguchi M, Ueda S (2005) Degeneration of monoaminergic fibers in the aged micrencephalic rat. Neurosci Lett 385:82-86

Zhou FC, Azmitia EC (1986) Induced homotypic sprouting of serotonergic fibers in hippocampus. II. An immunocytochemistry study. Brain Res $373: 337-348$ 